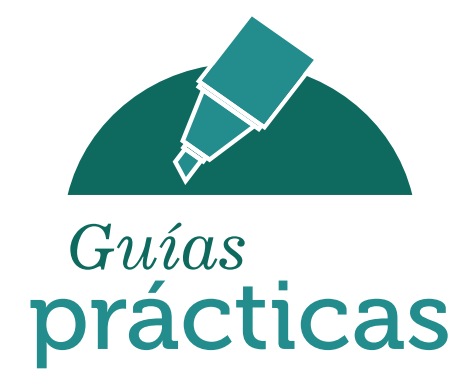

Apropiación social del conocimiento

Generación de contenidos impresos https://repository.ucc.edu.co/handle/20.500.12494/7375

\title{
ANATOMÍA ÓSEA PARA ESTUDIANTES
}

Álvaro José López Núñez

Universidad Cooperativa de Colombia

Sede Santa Marta 


\section{ACERCA DEL AUTOR}

Álvaro José López-Núñez, fisioterapeuta, especialista en docencia universitaria, maestrante en Ciencias de la Actividad Física y el Deporte, profesor auxiliar de la Facultad de Medicina de la Universidad Cooperativa de Colombia, sede Santa Marta.

Correo electrónico: alvaroj.lopez@campusucc. edu.co

\section{CÓMO CITAR ESTE DOCUMENTO}

López-Núñez AJ. Anatomía ósea para estudiantes (Generación de contenidos impresos N. ${ }^{\circ}$ 12). Ediciones Universidad Cooperativa de Colombia, 2021. DOI: https://doi.org/10.16925/ gcgp.34

NOTA LEGAL

El presente documento de trabajo ha sido incluido dentro de nuestro repositorio institucional como Apropiación social de conocimiento por solicitud del autor, con fines informativos, educativos o académicos. Asimismo, los argumentos, datos y análisis incluidos en el texto son responsabilidad absoluta del autor y no representan la opinión del Fondo Editorial o de la Universidad.

\section{DISCLAIMER}

This coursework paper has been uploaded to our institutional repository as Social Appropriation of Knowledge due to the request of the author. This document should be used for informational, educational or academic purposes only. Arguments, data and analysis included in this document represent authors' opinion not the Press or the University.

(c) (1) (-) Este documento puede ser consultado, descargado o reproducido desde nuestro repositorio institucional (http://repository. ucc.edu.co/handle/20.500.12494/7369) para uso de sus contenidos, bajo la licencia de Creative Commons Reconocimiento-NoComercial-SinObraDerivada 4.0 Internacional. http://creativecommons.org/licenses/by-nc-nd/4.0/ 


\section{TABLA DE CONTENIDO}

REGLAMENTO INTERNO DEL LABORATORIO DE MORFOLOGÍA

$\begin{array}{lr}\text { INTRODUCCIÓN } & 6\end{array}$

Propósitos de la práctica $\quad 6$

Recomendaciones prácticas de la guía 6

MARCO TEÓRICO

$\begin{array}{ll}1.1 \text { Sistema óseo } & 7\end{array}$

1.2 Esqueleto $\quad 7$

OBJETIVOS Y MATERIALES

2.1 Objetivos 8

2.2 Materiales 9

DESCRIPCIÓN DE ACTIVIDADES DE LA PRÁCTICA 9

EVALUACiÓN DE LA GUÍA DE PRÁCTICA DE ANATOMÍA ÓSEA IO

$\begin{array}{ll}\text { REFERENCIAS IO } & \text { IO }\end{array}$ 


\title{
12 ANATOMÍA ÓSEA PARA
}

\author{
Álvaro José López Núñez
}

\section{Resumen}

El hueso es un tejido conectivo duro, muy especializado que compone el esqueleto humano, aunque en el recién nacido son blandos y flexibles por su componente principal, los cartílagos. Según el tipo de tejido, se clasifican como compacto y esponjoso; y de acuerdo con su forma se denominan: largos, cortos, planos, irregulares y sesamoideos. Funcionalmente, el esqueleto se divide en axial, ubicado en la línea media del cuerpo humano y Apendicular, localizado por fuera del eje central del cuerpo. Sus funciones destacan el movimiento del cuerpo humano, protección de órganos internos, mantenimiento de la postura, almacén metabólico, sostenimiento del cuerpo humano y productor de células sanguíneas. Con esta guía se pretende que el estudiante del curso sistema músculo esquelético y tegumentario de la Facultad de Medicina identifique y realice la armazón del esqueleto en el laboratorio de morfología y refuerce el estudio anatómico del sistema óseo.

Palabras clave: anatomía ósea, esqueleto apendicular, esqueleto axial, hueso. 
Reglamento interno del laboratorio de morfología

1. La manipulación de modelos anatómicos, Osteoteca, Anatomage y material didáctico y orgánico del laboratorio debe hacerse con el mismo cuidado y respeto como se hace con un paciente.

2. El horario en el que funciona el laboratorio está establecido de lunes a viernes de 7 a.m. a 12 m y 2 p.m. a 6 p.m.

3. Las maletas, bolsos y cosas personales se deben dejar en los casilleros destinados para guardarlos.

4. Prohibido consumir alimentos y bebidas e, incluso, masticar gomas de mascar durante la práctica en el laboratorio. Tampoco se puede fumar.

5. Usar bata blanca mangas largas marcada con su nombre y logo de la universidad.

6. Utilizar elementos de bioseguridad durante la práctica y lavar las manos antes de su ingreso y a la salida de este.

7. Trabajar en silencio durante la práctica, mantener el orden en la sala y evitar usar bolígrafos sobre las estructuras anatómicas para no mancharlas.

8. Se restringe el uso de teléfonos móviles en las áreas de trabajo; a menos que el profesor o analista del laboratorio autorice su utilización por casos excepcionales.

9. El aforo máximo de cada sala del laboratorio no debe superar diez personas.

10. Los modelos anatómicos se le entregan al estudiante encargado de la mesa y debe ser devuelto en las mismas condiciones de limpieza.

11. Ningún equipo ni modelo anatómico, se podrá retirar del laboratorio de morfología.

12. El profesor encargado de la práctica debe entregar la guía del laboratorio al analista previamente del inicio de la sesión.

13. Al final de la práctica, el analista recoge la guía resuelta por el grupo de estudiantes.

14. La cancelación de una práctica se hace con anticipación.

15. Las normas consensuadas por los profesores y estudiantes se anotarán en un acta de laboratorio. 


\section{Introducción}

El componente de anatomía de los cursos por sistemas es una cátedra obligatoria en los programas de clase y de curso en los pregrados de Ciencias de la Salud. En la Facultad de Medicina, junto con la Embriología y la Histología, se encuentran los cursos de Morfología, que le brindan al estudiante los cimientos base de sus estudios médicos y, por ende, del desarrollo de su práctica profesional. La comprensión de los conceptos básicos en anatomía, la diferenciación entre la posición anatómica de referencia, los términos de posición, dirección y movimiento y las diversas connotaciones de la práctica en morfología apoyan al alumno en la construcción del conocimiento necesario para su formación como futuro profesional y minimiza errores en dicha práctica. Esta guía permite reforzar esos conocimientos y diferenciar el tipo de tejido óseo, clasificar el esqueleto, identificar los accidentes óseos e interrelacionar las estructuras óseas con las de otros sistemas del cuerpo humano, para así alcanzar las competencias necesarias durante la formación del estudiante.

\section{PROPÓSITOS DE LA PRÁCTICA}

El propósito de la presente práctica es identificar el esqueleto, afianzar terminología anatómica vista previamente en las actividades teóricas e interrelacionar otros conceptos de los componentes de fisiología y patología del sistema óseo que son revisados durante el desarrollo de esta práctica. La ejecución de las actividades durante la guía de práctica reforzará la perspectiva de la anatomía del sistema esquelético, con miras a la construcción de un nuevo conocimiento de los estudiantes.

\section{RECOMENDACIONES PRÁCTICAS DE LA GUÍA}

La presente guía está dirigida a estudiantes del primer semestre del programa de Medicina, matriculados en el curso Sistema Músculo Esquelético y Tegumentario y se ejecutará en una práctica de laboratorio. Se sugiere hacer una revisión teórica de la temática antes del desarrollo de esta, como también lo relacionado con el propósito y los objetivos de la práctica para facilitar su desarrollo [1].

Durante el proceso de la práctica y resolución de la guía, el estudiante seguirá los pasos establecidos para la comprensión del tema propuesto, que le servirá como base de conocimientos para adquirir la competencia del curso y apoyo del componente de anatomía de los otros cursos de sistemas. 


\section{MARCO TEÓRICO}

\subsection{SISTEMA ÓSEO}

El hueso es un tejido vivo provisto de tejido conectivo duro altamente especializado que compone la mayor parte del esqueleto del adulto. Los huesos del recién nacido son blandos y flexibles porque están compuestos principalmente por cartílagos. Los dos tipos de huesos son el compacto y el esponjoso, los cuáles se diferencian por la cantidad de material sólido y por el número y el tamaño de los espacios que contienen [2].

Según su forma, los huesos se clasifican como: largos, cortos, planos, irregulares y sesamoideos. Desde el punto de vista funcional, el sistema esquelético se divide en esqueleto axial y esqueleto apendicular [3]. Entre las funciones del sistema esquelético se destacan: movimiento, sostén mecánico, mantenimiento de la postura, protección de órganos internos, almacén metabólico y productor de células sanguíneas [4].

\subsection{ESQUELETO}

El esqueleto del ser humano está formado por 206 huesos, aunque en el lactante y en niños suman más de esa cifra, porque, algunos huesos, como los de la columna vertebral y los de la cadera, se fusionan más adelante [5]. Los huesos que se agrupan alrededor de la línea media del cuerpo suman 80 y hacen parte del esqueleto axial: cabeza ósea, los huesecillos auditivos, el hioides, el tórax óseo y la columna vertebral. Por otro lado, los huesos que se encuentran ubicados por fuera de la línea media suman 216 y constituyen el esqueleto apendicular, del cual hacen parte los huesos de los miembros superiores e inferiores, incluyendo las cinturas escapular y pélvica [6].

\subsubsection{Esqueleto axial}

El esqueleto de la cabeza está formado por 22 huesos que se agrupan en huesos del cráneo y huesos de la cara. El cráneo protege el encéfalo y sus huesos se dividen en pares e impares. Los huesos pares son los dos parietales y los dos temporales. Los huesos impares son: frontal, etmoides, esfenoides y occipital. En la cara, también se encuentran huesos pares; como: nasales, cigomáticos, maxilares superiores, lacrimales, cornetes inferiores y los palatinos; e impares como el vómer y el maxilar inferior.

El esqueleto del tórax está formado por el esternón y doce pares de costillas que se clasifican como: verdaderas (las 7 primeras), las cuáles se unen al esternón a través de un cartílago único. Las costillas octava, novena y décima se denominan falsas y se caracterizan por tener un cartílago común que las une al esternón y las costillas flotantes, que no se unen al esternón y son las dos últimas.

Las vértebras son los huesos de la columna vertebral que se encargan de proteger la medula espinal y se diferencian en regiones así: cervical (la más superior), conformada por 7 vértebras que se identifican por tener agujero transverso. Dorsal o torácica, ubicada en el dorso y constituida por 12 vértebras, caracterizadas por tener carillas articulares para inserción costal; y lumbar, conformadas por 5 vértebras que presentan un cuerpo vertebral más grande al compararlas con las otras regiones y no tener agujero transverso ni carillas costales. La porción distal de la columna vertebral presenta los huesos sacro y coxis, que resultan de la fusión de las vértebras del mismo nombre. 


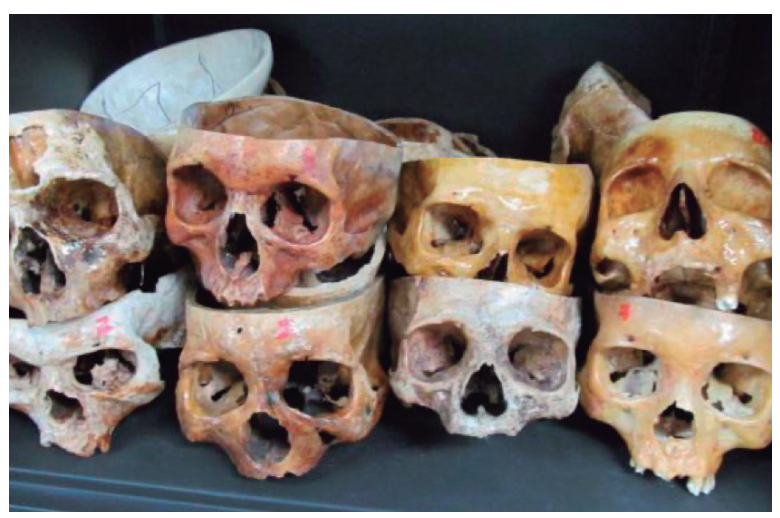

FIGURA 1. Estructuras del esqueleto. Axial (cráneos) Fuente: elaboración propia.

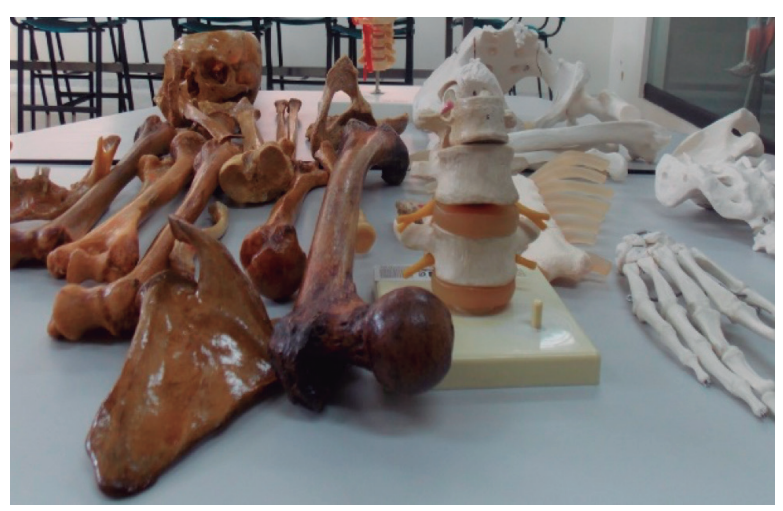

FIGURA 2. Algunos huesos de esqueleto apendicular óseos Fuente: elaboración propia.

\subsubsection{Esqueleto apendicular}

Los huesos de los miembros también se pueden estudiar por regiones. La cintura escapular está constituida por las clavículas (2) y las escápulas (2). Cada extremidad superior tiene 30 huesos localizados en tres regiones. El brazo, tiene un hueso llamado húmero. En el antebrazo se encuentran el radio, lateralmente y el cúbito, medialmente. La mano está conformada por ocho huesos en el carpo, cinco en el metacarpo y catorce en los dedos (falanges). La cintura pélvica, tiene dos huesos llamados coxales; uno en cada cadera. De la misma manera que la extremidad superior, la inferior también tiene 30 huesos en cada miembro. El hueso del muslo es conocido como fémur y es el más grande del ser humano. Anterior a la rodilla se encuentra la patela, hueso sesamoideo muy importante para la función y la estabilización de dicha articulación. En la pierna, están la tibia medialmente y el peroné, lateralmente. En el pie, se observan siete huesos en el tarso, cinco en el metatarso y catorce en los dedos [7].

\section{OBJETIVOS Y MATERIALES}

\subsection{OBJETIVOS}

\subsubsection{Objetivo general}

Identificar el esqueleto, afianzando la terminología anatómica vista previamente en las actividades teóricas e interrelacionar otros conceptos de los componentes de fisiología y patología del sistema óseo que son revisados durante el desarrollo de dicha práctica.

\subsubsection{Objetivos específicos}

- Diferenciar el esqueleto axial del apendicular en cuanto a su forma, estructura, función e interrelaciones con las regiones del cuerpo humano.

- Clasificar los componentes anatómicos del esqueleto.

- Explicar la estructura anatómica del esqueleto.

- Realizar asociaciones entre los componentes morfológicos y fisiológicos del sistema óseo.

- Aplicar conceptos sobre el daño estructural macroscópico del sistema óseo.

- Armar el esqueleto con las estructuras de la Osteoteca del laboratorio de morfología en la práctica de anatomía ósea. 


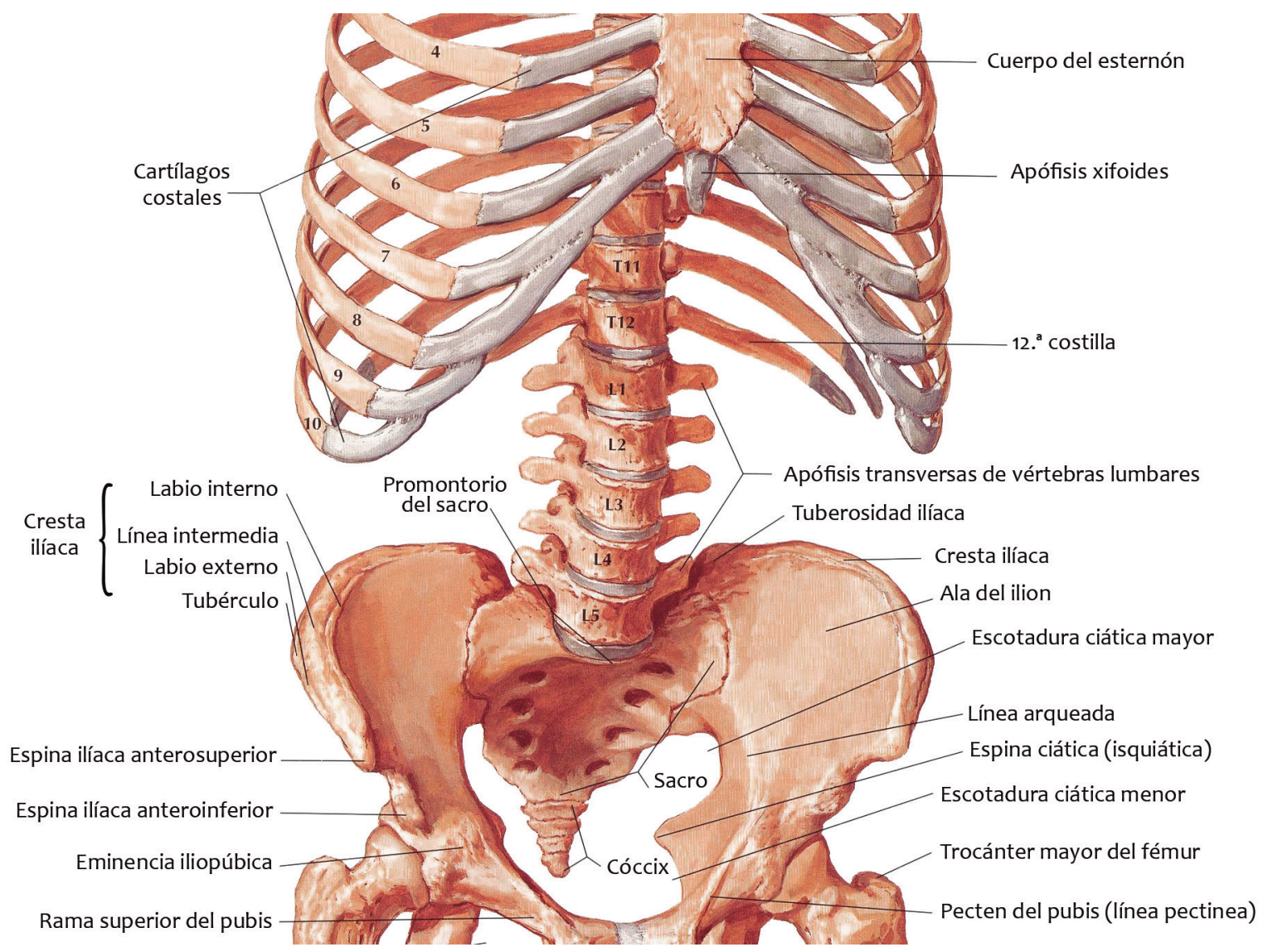

FIGURA 3. Tórax y pelvis

Fuente: [8]

\subsection{MATERIALES}

- Guantes de nitrilo.

- Gorro quirúrgico.

- Bata marcada.

- Tapabocas.

- Alfileres para señalar.

- Modelos anatómicos óseos.

- Elementos óseos de la Osteoteca.

- Tableta Anatomage.

- Cuaderno de notas.

- Tablero, marcadores y ayudas audiovisuales.
DESCRIPCIÓN DE ACTIVIDADES DE LA PRÁCTICA

Seguidamente, se describe un paso a paso para la realización de la práctica de anatomía ósea, reconocimiento de las estructuras, identificación de los huesos y su lateralidad y construcción o armado del esqueleto en la mesa de disección por grupos de seis estudiantes.

- El alumno representante de cada grupo se dirigirá a la Osteoteca para que el analista del laboratorio le haga entrega de los huesos requeridos para la práctica.

- Los estudiantes manipularán con guantes de látex, gorro, batas y tapabocas los huesos orgánicos y sintéticos por normas de bioseguridad. 
- El profesor asesor de la práctica apoyará, durante el tiempo que esta dure; además de realizar asesorías en la tableta Anatomage.

- Después de armar el esqueleto, los estudiantes de cada mesa de disección sustentarán la construcción del esqueleto basados en el soporte teórico.

- Los estudiantes tendrán en cuenta las regiones anatómicas, la dirección de las estructuras y la posición de los huesos para poder construir su esqueleto en la mesa de actividad.

- Al final de la práctica, cada estudiante por mesa redactará su informe final en el que resaltarán las conclusiones.

\section{EVALUACIÓN DE LA GUÍA DE PRÁCTICA DE ANATOMÍA ÓSEA}

Inmediatamente después de la realización de la práctica de anatomía ósea, el alumno presentará un informe con el registro de la experiencia en el que se demuestra la competencia del estudiante. El informe se debe presentar de acuerdo con las siguientes directrices.

- Portada. Es la primera página del informe en la que se incluye el nombre de la universidad, facultad, curso, grupo, nombre de la práctica, nombre del estudiante y fecha de ejecución de la actividad.
- Introducción. En este apartado el estudiante describe el fundamento teórico de la práctica y los objetivos de la actividad. Debe existir correlación entre ellos; además, de ser medibles afines a los resultados esperados.

- Materiales y métodos. En este párrafo, el alumno destaca los materiales utilizados para la realización de la práctica y describe brevemente cómo es la posición de los huesos por regiones para armar el esqueleto.

- Resultados. En esta sección, el alumno describe los resultados de la práctica; en esta pueden utilizarse mapas conceptuales o cuadros sinópticos sobre la referencia de la práctica. Las sugerencias del estudiante acerca de la práctica también son importantes como realimentación para mejora de esta.

- Discusión. Aparte de presentarse el análisis de los resultados de la práctica en este ítem se presentan las conclusiones y las recomendaciones para mejora de la práctica.

- Referencias bibliográficas. Esta parte es para que el estudiante consigne las referencias y fuentes de consulta del trabajo; teniendo en cuenta usar las referencias usadas en la práctica y en la redacción de su informe.

\section{REFERENCIAS}

[1] Neiza-Rodríguez N. Guía de práctica de anatomía del corazón dirigida a estudiantes. (Documento de docencia n. 28). Bogotá: Ediciones Universidad Cooperativa de Colombia, 2017. Doi: https://doi.org/10.16925/ greylit.2281

[2] Pineda D. et al. Anatomía: Manual de actividades para el autoaprendizaje. 1ª edición. Bogotá: Editorial Médica Internacional; 2021.

[3] Pró E. Anatomía clínica. 2ª ed. Buenos Aires: Editorial Médica Panamericana; 2014.

[4] Tórtora G, Derrickson B. Principios de anatomía y fisiología. 13ª ed. México: Editorial Médica Panamericana; 2013. 
[5] Moore KL. Anatomía con orientación clínica. 8ª ed. Barcelona: Wolters Kluwer; 2017.

[6] Drake R. et al. Gray. Anatomía para estudiantes. 4a ed. Barcelona: Elsevier; 2016.

[7] Hall S. et al. Lo esencial en Anatomía y Fisiología. 5ª ed. Barcelona: Elsevier; 2019.

[8] Netter F. Atlas de anatomía humana 7ª ed. España: Elsevier; 2019. 


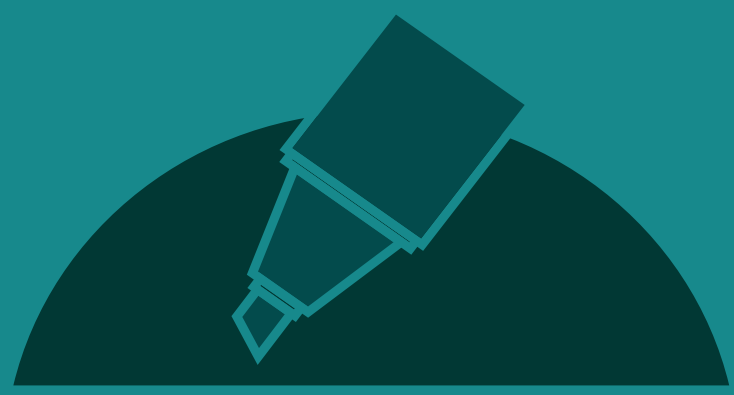

Guías prácticas 